\title{
The Indolent Nature of Primary Mucinous Carcinoma of the Ovary
}

\author{
Jessica M. Gillen MD', Sanam Husain $\mathrm{MD}^{2}$, Kai Ding $\mathrm{PhD}^{3}$, Kathleen N. Moore MD${ }^{1}$, \\ Camille C. Gunderson $\mathrm{MD}^{1^{*}}$ \\ ${ }^{1}$ Section of Gynecologic Oncology, Department of Obstetrics and Gynecology, \\ University of Oklahoma, Oklahoma City, OK, USA \\ ${ }^{2}$ Department of Pathology, University of Oklahoma, Oklahoma City, OK, USA \\ ${ }^{3}$ Department of Biostatistics and Epidemiology, University of Oklahoma, Oklahoma City, OK, USA \\ ${ }^{1 *}$ camille-gunderson@ouhsc.edu
}

\begin{abstract}
:
Objective: Mucinous epithelial ovarian carcinoma (mEOC) is rare and has a distinct presentation, disease distribution, and response to treatment versus serous cancers. We sought to further support the contrast between these histologies by quantifying occult intraperitoneal metastases, isolated lymph node metastasis, and characterization of whether invasive subtype may influence clinical outcomes.

Methods: We performed a retrospective review of patients with mEOC at The University of Oklahoma from 1996-2014 and recorded clinicopathologic data. Pathologic re-analysis was performed whenever possible. SAS version 9.4 was used for analysis.

Results: 46 patients met criteria, the majority of which (78.3\%) had stage I disease. Of these, $48.7 \%$ received adjuvant platinum-based chemotherapy. Occult metastatic disease was identified in 1 patient; none had isolated nodal metastasis. Of the 7 patients who underwent reoperation for staging of apparent stage I mEOC, 1 had occult metastasis. Six patients had tumors arising from non-ovarian/unknown location on pathologic rereview. Invasive patterns were $67 \%$ expansile and $33 \%$ infiltrative; $16 \%$ with infiltrative pattern recurred, and $8.3 \%$ with expansile subtype recurred. At year 1, 2 and 3,97.4\%, 94.5\% and $86.1 \%$ of patients remained alive, respectively.

Conclusion: Occult peritoneal or lymph node metastasis is exceedingly rare with mEOC. Rare tumors such as this merit research in aggregate in order to synthesize better therapeutic modalities. Although most women have early stage disease and will not recur, those who do have a very poor prognosis given the dearth of agents with activity against this aggressive disease subtype at present.
\end{abstract}

Keywords: mucinous, ovarian cancer, expansile, infiltrative.

\section{INTRODUCTION}

Primary mucinous ovarian cancer is rare and accounts for $<5 \%$ of the 21,290 new ovarian cancers per year in the United States.[1] It has a clinically distinct presentation and response in comparison to its serous counterparts, which account for most epithelial ovarian cancers (EOC).[2] These two subtypes have traditionally been treated uniformly; however, many authors suggest that varying histologies of EOC should be considered different diseases with distinct treatment paradigms.[3]

Both pathologic features and genetic signatures support a fundamental difference between these tumors. Invasive mucinous adenocarcinoma may be pathologically defined as either expansile or infiltrative. The expansile subtype is characterized by confluent glandular growth that is uninterrupted by normal ovarian tissue, while infiltrative invasion is defined by small glands or nests of cells invading the stroma. Infiltrative growth is more aggressive than expansile invasion [3-5] and is correlated with poor prognosis in colorectal cancer. A suggestion of poorer outcomes has been demonstrated with mucinous EOC, but data is limited to date.[4] Molecular signatures also differ; approximately $50 \%$ of mucinous cancers have a KRAS mutation, as compared to only $5 \%$ of serous EOC. Additionally, BRCA1, BRCA2, and p53 mutations, which are thought to play an integral role in the development of serous cancers of the ovary, have demonstrated little to no association with mucinous tumors. $[6,7]$ 
Mucinous EOC has a distinct clinical presentation and course. Across all histologic classifications, most women with EOC will be diagnosed with advanced stage disease. While this is typical with serous adenocarcinomas, most women with mucinous EOC will actually be stage I wherein 5-year survival rates are excellent (approaching 90\%). Unfortunately, the same cannot be said with advanced stage disease. Hess et al demonstrated that women with stage III/IV mucinous EOC had a progression free survival (PFS) of only 5.7 months, compared to 14.1 months with other epithelial histologies. Overall survival (OS) was also substantially shorter (12.0 months as compared to 36.7 months).[2] This was further supported in an ancillary review of 6 phase III Gynecologic Oncology Group trials of women with stage III EOC receiving adjuvant chemotherapy; women with mucinous EOC had a median OS of only 14.8 months versus 45.2 months in women with serous carcinoma.[8]

The dichotomy in outcomes is likely multifactorial. Mucinous EOC seems to be inherently platinum resistant, with response rates of $12.5-38.5 \%$ versus $67.7-70 \%$ in serous cancers. [9] This disparity has prompted exploration of alternative treatment strategies for mucinous EOC utilizing a gastrointestinal regimen such as oxaliplatin and capecitabine in Gynecologic Oncology Group protocol 241 (NCT 01081262).[10] Furthermore, occult metastasis in patients with clinically stage I mucinous EOC occurs infrequently, and it has been demonstrated that isolated nodal metastases do not occur when tumors appear confined to the ovary.[6, 7, 11] Conversely, with clinically stage I serous cancers, occult nodal metastasis is found in $10 \%$ of patients.[12] In this study, we aim to further support the distinction between these histologies by determining the incidence of occult metastasis and isolated lymph node metastasis in patients with clinical stage I primary mucinous EOC. We also seek to further characterize how histologic subtype (expansile or infiltrative) influences clinical outcomes.

\section{Materials AND Methods}

This is an IRB-approved (study \#3600) retrospective review of patients who underwent treatment for EOC at The University of Oklahoma from 1996-2014. Women with histology proven, invasive mucinous EOC were included. Women who underwent surgery elsewhere had central pathology review confirming diagnosis. Patients with benign or low malignant potential mucinous tumors were excluded, as were those with mucinous tumors of GI primary. For each case, demographics and pathologic information including stage, preoperative CA125 and CEA, and surgical procedure (primary debulking vs interval) were collected. Additional variables considered included presence of occult or nodal metastasis, chemotherapy regimens, disease recurrence, and dates of follow-up. All cases were reviewed at a multidisciplinary tumor board with gynecologic pathologists and gynecologic oncologists for confirmation of diagnosis and treatment recommendations. All slides were re-reviewed (when available) by a gynecologic pathologist specifically for this study.

Among the 18 mucinous neoplasms re-reviewed, 5 tumors were of non-ovarian types. The remaining tumors had gross and histologic features consistent with ovarian origin. The morphologic patterns of invasion were evaluated in these cases and categorized as expansile or infiltrative subtypes of stromal invasion. Expansile stromal invasion was defined by exuberant glandular proliferation resulting in the formation of cribriform or papillary architecture with little to no intervening stroma. Additionally, prominent glandular branching with obliteration of surrounding stromal tissue also qualified as expansile invasion. The focus of glandular confluence had to exceed $5 \mathrm{~mm}$ in area to meet criteria for invasion. Infiltrative stromal invasion was categorized by nests and cords of tumor cells arranged infiltrating the stroma with jagged outlines or overtly malignant single cells with associated desmoplasia. One of the tumors demonstrated microinvasion defined by a focus of tumor cells invading the stroma less than $5 \mathrm{~mm}$ in greatest dimension. All tumors with an invasive component were accompanied by a bona fide mucinous borderline component, and all exhibited intestinal differentiation. Mucinous carcinomas were graded using the FIGO grading system based on architectural and nuclear grade.

Median (with range) was used to summarize continuous variables. Count (percentage) was used to summarize categorical data. Comparisons of percentages between two groups were based on Fisher's exact test. Outcomes evaluated included progression free survival (PFS) and overall survival (OS). PFS was defined as time from completion of primary chemotherapy to first recurrence based on RECIST or CA125 criteria (or date of last follow-up if patient did not experience recurrence). OS was characterized as time from diagnosis to death (or date of last follow-up if still living at time of data collection). Event (disease progression or death)-free rates at specified time points were estimated based on the Kaplan-Meier method. All statistical analyses were performed using SAS version 9.3 (SAS Institute; Cary, NC) 


\section{Results}

Between 1996 -2014, 592 women underwent evaluation for EOC at our institution. Of these, 46 had primary invasive mucinous EOC and thus met inclusion criteria. The average age at diagnosis was 48.6 years (range, 16-85). Median CA125 and CEA values at diagnosis were 54 units $/ \mathrm{mL}$ (range, 9.81352 ) and $2.5 \mathrm{ng} / \mathrm{mL}$ (range, 0.6-35.2), respectively. The majority of patients (78.3\%) had stage I disease, and of those with known grade, $42.5 \%$ were well differentiated (see Table 1). All but one patient underwent primary surgery (deemed ineligible for surgery due to pulmonary emboli), and most (96\%) were left with no gross residual disease. Only 2 patients (both were stage IIIC) required radical procedures for debulking, including small bowel and colon resection. Occult metastatic disease was identified in one patient (microscopic disease on staging biopsies of the peritoneum). No patients had isolated nodal metastases (see Table 2). Seven patients underwent reoperation after primary mucinous carcinoma of the ovary was unexpectedly noted on final pathology; of these, one had evidence of disease upon laparotomy.

Table1. Subject demographics

\begin{tabular}{|c|c|}
\hline Characteristic & Number of subjects (\%) \\
\hline Age, median (range) & $46(16-85)$ \\
\hline Stage & $36(78 \%)$ \\
I & $5(11 \%)$ \\
II & $5(11 \%)$ \\
III & $0(0 \%)$ \\
IV & $17(37 \%)$ \\
Grade & $14(30.4 \%)$ \\
2 & $9(19.6 \%)$ \\
3 & $6(13 \%)$ \\
Unknown & $2(4 \%)$ \\
Yes & $44(96 \%)$ \\
No & $22(47.8 \%)$ \\
\hline Residual disease after surgery & $24(52.2 \%)$ \\
\hline Platinum based & \\
\hline
\end{tabular}

Table2. Metastatic sites at original surgery

\begin{tabular}{|c|c|}
\hline Site & Number of patients (\%) \\
\hline Isolated Nodal Metastasis & $0(0 \%)$ \\
\hline Diaphragm & $0(0 \%)$ \\
\hline Spleen & $0(0 \%)$ \\
\hline Small Bowel & $2(4.4 \%)$ \\
\hline Colon & $2(4.4 \%)$ \\
\hline Peritoneum & $1(2.2 \%)$ \\
\hline
\end{tabular}

Specimens from 18 patients were available for pathologic reanalysis to determine organ of origin and pattern of invasion. Four patients were deemed to have tumor arising from non-ovarian origin; 3 were gastrointestinal (GI), 1 breast, and 2 unknown/other. Of the mucinous EOCs, invasive patterns were 67\% expansile (see Figure 1) and 33\% infiltrative (see Table 3; see Figure 2). When evaluating disease recurrence by invasive subtype, $16 \%$ of those with infiltrative pattern recurred, and $8.3 \%$ with expansile invasion experienced disease recurrence $(\mathrm{p}=\mathrm{NS})$. The patients who recurred in both groups went on to die of disease.

Of the 41 patients with early stage disease, $48.7 \%$ received adjuvant therapy with platinum based chemotherapy, while $80 \%$ of those with advanced disease $(n=5)$ received cytotoxic chemotherapy $(\mathrm{p}=0.35)$. One patient did not receive adjuvant chemotherapy because re-review of her specimen suggested pseudomyxoma peritonei. Seventeen percent of early stage patients experienced recurrence, whereas $60 \%$ of those with advanced stage disease recurred $(\mathrm{p}=0.06)$. No patients with early stage disease who recurred were salvaged, as 5 died of disease and 2 are alive with disease burden. Median follow-up period was 25.2 months (range, 1.7-88.3). Neither median PFS nor OS could be established as $>50 \%$ of patients remained event-free at the time of analysis. At year 1, 2 and 3, 97.4\%, 94.5\% and $86.1 \%$ of patients remained alive respectively, and only 8 patients died of their disease, $94.5 \%$ of whom survived beyond 24 months. The overall disease recurrence rate was $21.7 \%$ with $90 \%, 84.6 \%$ and $73.9 \%$ of patients remaining progression free at month 12,18 and 24 respectively. 


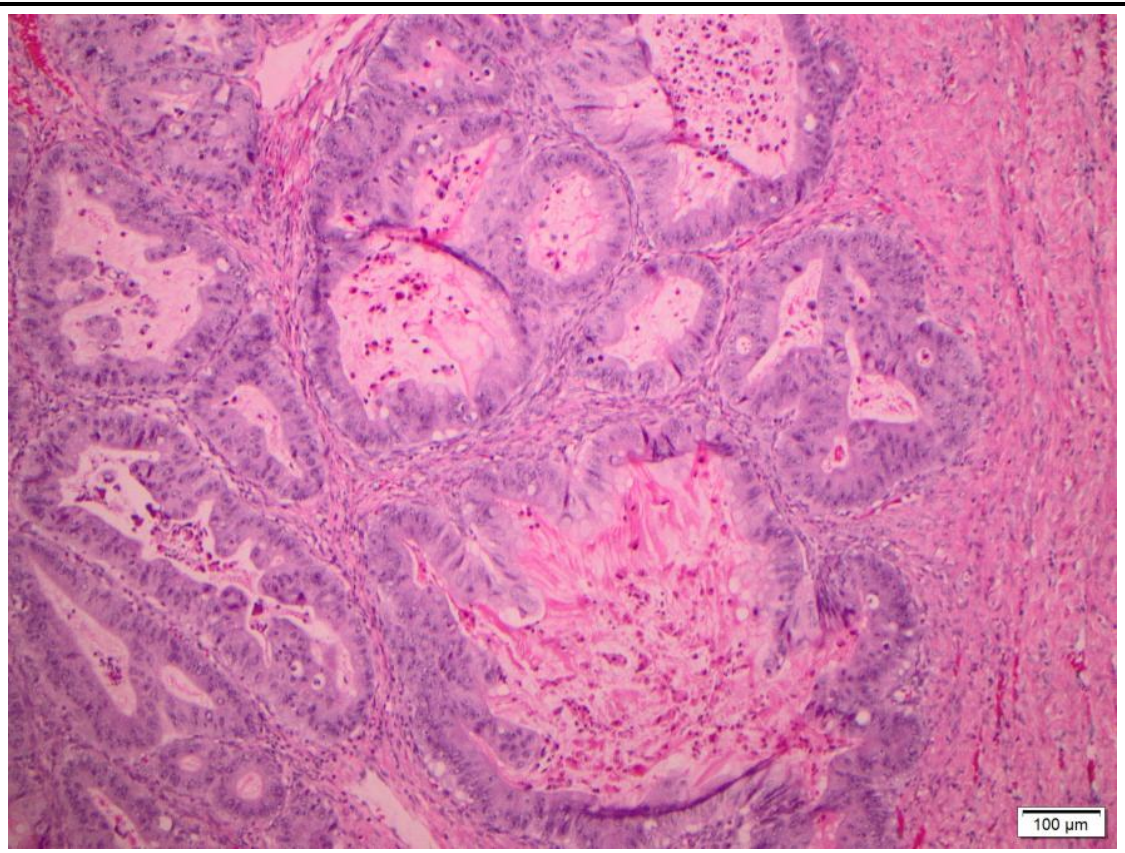

Figure1. Mucinous ovarian cancer, expansile invasion subtype

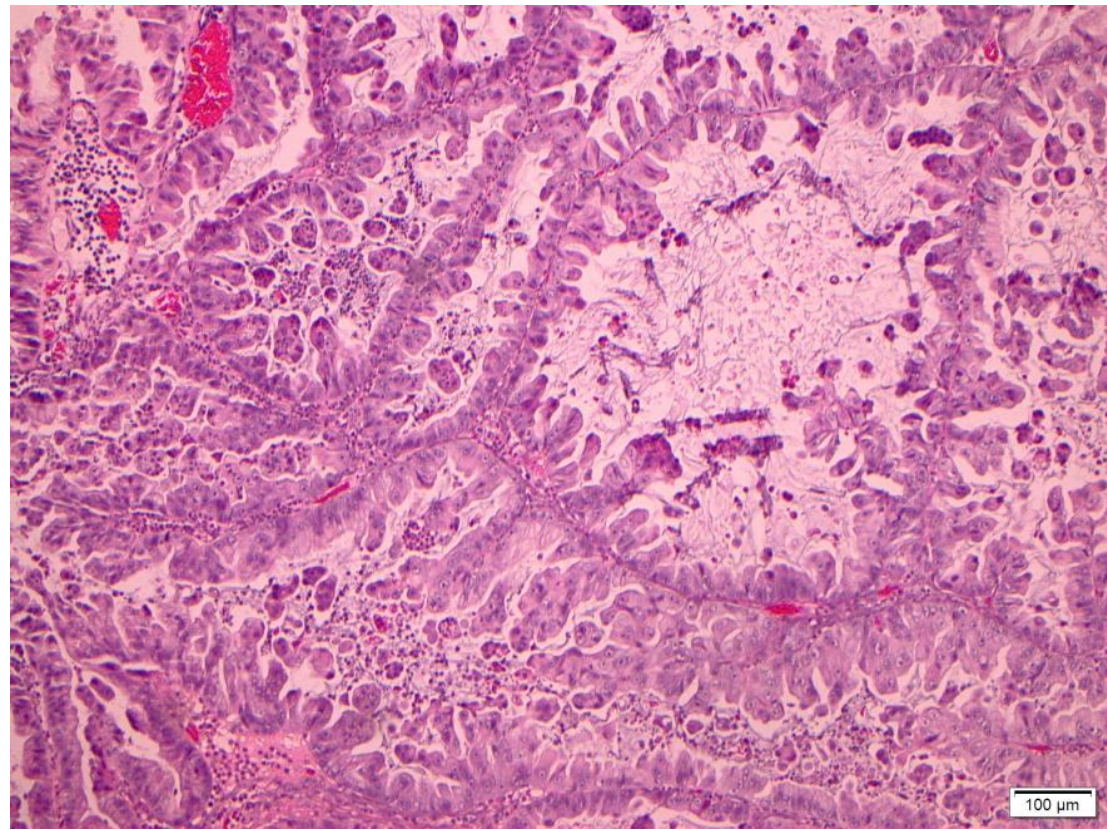

Figure2. Mucinous ovarian cancer, infiltrative invasion subtype

Table3. Pathologic features

\begin{tabular}{|c|c|}
\hline Pattern & Number of specimens (\%) \\
\hline Expansile & $12(66.7 \%)$ \\
\hline Infiltrative & $4(22.2 \%)$ \\
\hline Infiltrative, focally & $2(11.1 \%)$ \\
\hline Re-analysis of primary site & $3(16.7 \%)$ \\
\hline Gastrointestinal primary & $1(5.6 \%)$ \\
\hline Breast primary & $2(11.2 \%)$ \\
\hline Other primary/unknown & \\
\hline
\end{tabular}

\section{DISCUSSION}

Mucinous EOC has historically been treated similarly to all other EOC subtypes despite continued research demonstrating molecular distinctions and a more indolent clinical course for early stage disease. Our data supports previous findings that occult metastatic disease with clinically stage I mucinous EOC is infrequently discovered, as we noted this in only 1 patient. Additionally, we did not find any isolated nodal metastases, bolstering previous reports that this occurs with exceeding 
rarity. $[6,11,13]$ This information brings into question the utility of lymphadenectomy in this group of women, in particular when re-operation is being considered for those with incidentally noted mucinous EOC which was visibly confined to the ovary.

Although most patients with mucinous EOC fortunately have an indolent disease course, the subset of patients who present with advanced or recurrent disease are typically chemoresistant and die of disease. Advanced or recurrent mucinous EOC behaves dissimilar to most ovarian cancers and may be more analogous to aggressive gastrointestinal tract cancers, which carry a very grim prognosis. In fact, it can be difficult to pathologically differentiate mucinous EOC from mucinous tumors of GI or other primary. Of the 18 cases in our series with pathology available for re-analysis, 4 patients had tumors not confirmed to be mucinous EOC. Three were thought to originate from the GI tract and 1 from breast; 2 were unable to be determined. The disparate results of pathologic re-analysis are similar to those reported in GOG 241, in which specialist pathology review demonstrated non-ovarian primary in 19/36 cases. This striking difference suggests that perhaps all mucinous EOC should undergo consensus review to confirm proper diagnosis. In terms of survival, our results align with others who have reported OS of 15 months for this poor prognostic group.[14] This further underscores the clinical dichotomy of patients with mucinous EOC and the need for targeted therapies for the patients with traditionally chemoresistant and lethal disease.

The indolent or aggressive nature of mucinous EOC may not only depend on stage, but also on pathologic type of invasion. Our data show a trend towards more aggressive disease in those patients with infiltrative disease as compared to those with expansile invasion; however, the small number of patients precludes robust comparisons. While few studies have shown marginal differences in prognosis and recurrence between these pathologic findings, our findings are in keeping with previous data that showed a more aggressive clinical course when infiltrative invasion was clearly present.[15] We recommend routine categorization of this rare tumor type by invasive subtype to facilitate further study of this component.

There are several notable limitations of our study. First, the retrospective nature lends to incomplete medical records and inevitable loss to follow-up for a fraction of patients. Second, some tumors may have originated from the gastrointestinal tract and other sites given difficulty in pathologic characterization to confirm organ of origin in some cases, similar to the experience reported by others. Third, only about $1 / 3$ of patients had an invasive subtype (expansile versus infiltrative) detailed, and thus evaluation of the association of this parameter with clinical outcome was limited.

In conclusion, rare tumors such as mucinous EOC merit research in aggregate in order to synthesize better therapeutic modalities. Although most women will have early stage disease and will not experience disease recurrence, those who recur have a poor prognosis given the dearth of agents with activity against this disease type at present. For women with apparent early stage disease, our data suggests that reoperation for staging is very low yield given the infrequency of occult metastatic disease and lack of nodal metastasis with mucinous EOC. For patients with advanced or recurrent disease, avoidance of conventional chemotherapy with emphasis on individualized therapies based on somatic profiling and embracement of palliative care when these are not available may be more appropriate.

\section{Conflict of Interest:}

Dr. Moore acknowledges advisory board compensation from AstraZeneca, Amgen, Genentech/Roche, Immunogen, VBL Pharmaceuticals and Clovis. These relationships have no relevance to this submitted work. Drs. Gunderson, Gillen, Husain and Ding report no conflicts.

\section{REFERENCES}

[1] Siegel, R.L., K.D. MillerA. Jemal Cancer statistics, 2015. CA Cancer J Clin, 2015. 651: 5-29.

[2] Hess, V., et al. Mucinous epithelial ovarian cancer: a separate entity requiring specific treatment. J Clin Oncol, 2004. 226: 1040-4.

[3] Frumovitz, M., et al. Unmasking the complexities of mucinous ovarian carcinoma. Gynecol Oncol, 2010. 1173: 491-6.

[4] Lee, K.R.R.E. Scully Mucinous tumors of the ovary: a clinicopathologic study of 196 borderline tumors (of intestinal type) and carcinomas, including an evaluation of 11 cases with 'pseudomyxoma peritonei'. Am J Surg Pathol, 2000. 2411: 1447-64. 
[5] Rodriguez, I.M.J. Prat Mucinous tumors of the ovary: a clinicopathologic analysis of 75 borderline tumors (of intestinal type) and carcinomas. Am J Surg Pathol, 2002. 262: 139-52.

[6] Cho, Y.H., et al. Is complete surgical staging necessary in patients with stage I mucinous epithelial ovarian tumors? Gynecol Oncol, 2006. 1033: 878-82.

[7] Morice, P., et al. Lymph node involvement in epithelial ovarian cancer: analysis of 276 pelvic and paraaortic lymphadenectomies and surgical implications. J Am Coll Surg, 2003. 1972: 198205.

[8] Winter, W.E., 3rd, et al. Prognostic factors for stage III epithelial ovarian cancer: a Gynecologic Oncology Group Study. J Clin Oncol, 2007. 2524: 3621-7.

[9] Pectasides, D., et al. Advanced stage mucinous epithelial ovarian cancer: the Hellenic Cooperative Oncology Group experience. Gynecol Oncol, 2005. 972: 436-41.

[10] Gore ME, H.A., Bradey WE, Penson RT, Zaino RJ et al. Multicentre trial of carboplatin/paclitaxel versus oxaliplatin/capecitabine, each with/without bevacizumab, as first line chemotherapy for patients with mucinous epithelial ovarian cancer (mEOC). . J Clin Oncol, 2015. 33 suppl; abstr 5528.

[11] Schmeler, K.M., et al. Prevalence of lymph node metastasis in primary mucinous carcinoma of the ovary. Obstet Gynecol, 2010. 1162 Pt 1: 269-73.

[12] Leblanc, E., et al. Laparoscopic restaging of early stage invasive adnexal tumors: a 10-year experience. Gynecol Oncol, 2004. 943: 624-9.

[13] Roger, N., et al. Should pelvic and para-aortic lymphadenectomy be different depending on histological subtype in epithelial ovarian cancer? Ann Surg Oncol, 2008. 151: 333-8.

[14] Mackay, H.J., et al. Prognostic relevance of uncommon ovarian histology in women with stage III/IV epithelial ovarian cancer. Int J Gynecol Cancer, 2010. 206: 945-52.

[15] Tabrizi, A.D., et al. Primary ovarian mucinous carcinoma of intestinal type: significance of pattern of invasion and immunohistochemical expression profile in a series of 31 cases. Int $\mathbf{J}$ Gynecol Pathol, 2010. 292: 99-107. 